\title{
Time-Dependent Effects of Systemic Lipopolysaccharide Injection on Regulators of Antioxidant Defence Nrf2 and PGC-1a in the Neonatal Rat Brain
}

\author{
Fernando Correa $^{\mathrm{a}}$ Elin Ljunggren ${ }^{\mathrm{a}}$ Jaspal Patil ${ }^{\mathrm{a}}$ Xiaoyang Wang ${ }^{\mathrm{b}}$ \\ Henrik Hagberg $^{\mathrm{b}}$ Carina Mallard $^{\mathrm{b}}$ Mats Sandberg ${ }^{\mathrm{a}}$

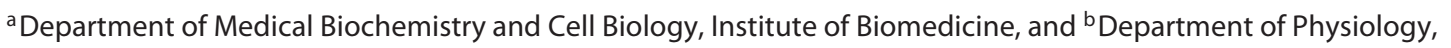 \\ Institute of Neuroscience and Physiology, University of Gothenburg, Gothenburg, Sweden
}

\section{Key Words \\ Neuroinflammation · Nuclear factor E2-related factor 2 . \\ Antioxidant system · Peroxisome proliferator-activated \\ receptor- $\gamma$ coactivator $1 a$}

\begin{abstract}
Background/Aims: Both excitotoxicity and neuroinflammation are associated with oxidative stress. One transcription factor, nuclear factor E2-related factor 2 (Nrf2), and one transcription cofactor, peroxisome proliferator-activated receptor- $\gamma$ coactivator $1 a$ (PGC-1a), increase the endogenous antioxidant defence and can thus modulate neuronal cell death. Here, we investigated the temporal effects (after 24 and 72 h) of systemic (i.p.) administration of lipopolysaccharide (LPS) on the cerebral Nrf2 and PGC-1a systems. Methods and Results: Seven-day-old rat pups were injected with LPS $(0.3 \mathrm{mg} / \mathrm{kg})$. After $24 \mathrm{~h}$, the protein levels of $\gamma$-glutamylcysteine ligase modulatory subunit, $\gamma$-glutamylcysteine ligase catalytic subunit, Nrf2, PGC-1a and manganese superoxide dismutase (MnSOD) were increased in parallel with decreased levels of Keap1. These effects were correlated with an increased level of phosphorylated Akt and elevated acetylation of histone 4 . In contrast, $72 \mathrm{~h}$ following LPS, a decrease in the components of the Nrf2 system in par-
\end{abstract}

allel with an increase in Keap1 was observed. The down-regulation after $72 \mathrm{~h}$ correlated with phosphorylation of p38 mitogen-activated protein kinase, while there were no changes in PGC-1 $a$ and MnSOD protein levels or the acetylation/methylation pattern of histones. Conclusion: Systemic LPS in neonatal rats induced time-dependent changes in brain Nrf2 and PGC-1a that correlated well with the protective effect observed after $24 \mathrm{~h}$ (pre-conditioning) and the deleterious effects observed after $72 \mathrm{~h}$ (sensitizing) of systemic LPS reported earlier. Collectively, the results point towards Nrf2 and PGC-1a as a possible mechanism behind these effects.

Copyright $\odot 2013$ S. Karger AG, Basel

\section{Introduction}

Excitotoxicity, oxidative stress and inflammation are tightly interrelated factors in the neurotoxic process in acute ischaemia and chronic neurodegenerative diseases $[1,2]$. Down-regulation of anti-oxidant systems promotes oxidative stress and inflammation caused by excitotoxicity [3]. The transcription factor nuclear factor E2-related factor 2 (Nrf2), a member of the 'cap ' $n$ ' collar' group of transcription factors, is essential for protecting cells

\section{KARGER}

E-Mail karger@karger.com

www.karger.com/nim
(C) 2013 S. Karger AG, Basel

1021-7401/13/0204-0185\$38.00/0
Fernando Correa

Department of Medical Biochemistry and Cell Biology Institute of Biomedicine, University of Gothenburg PO Box 420, SE-405 30 Gothenburg (Sweden)

E-Mail Fernando.correa@medkem.gu.se 
against oxidative damage [4]. Deletion of Nrf2 makes animals over-sensitive to oxidative stress and they develop white matter damage and retinopathy spontaneously [3, $5,6]$. Interestingly, brains from Alzheimer patients demonstrate low levels of Nrf2 in hippocampal astrocytes [7], indicating inadequate astroglial anti-oxidant support in this disease. Studies done in Nrf2 knockout mice show larger infarct volume after cerebral ischaemia, and tertbutylhydroquinone, an $\mathrm{Nrf} 2$ activator, limited the damage in the penumbra region but not in the core of the infarction area in wild-type animals only [8]. Similarly, the Nrf2 inducers sulphoraphane, curcumin and (-)-epicatechin have shown protective effects after transient middle cerebral artery (MCA) occlusion [9-11]. The endogenous induction of the Nrf2 system in the peri-infarct region [8] is, at least partly, likely due to down-regulation of the cytoplasmatic protein Keap1 that sequesters Nrf2 [12]. Regulation of the inducible anti-oxidant system in the neonatal central nervous system has not been studied in great detail; however, it has been shown that the Nrf2 activator sulphoraphane can protect against neonatal hypoxia-ischaemia in rats [13]. Our earlier studies showed that the level of glutathione (GSH) was decreased in brain after hypoxia-ischaemia [14]. In a later study, we demonstrated that the reduction in GSH levels was more pronounced when hypoxia-ischaemia was preceded by inflammation induced by lipopolysaccharide (LPS) injection in post-natal day 8 rats $72 \mathrm{~h}$ prior to the insult [15]. Both the brain damage and the decrease in GSH were normalized by multiple injections of the anti-oxidant and GSH precursor Nacetylcysteine, indicating that GSH synthesis was reduced by the inflammation [15]. In contrast, LPS injection $24 \mathrm{~h}$ prior to hypoxia-ischaemia protected against brain damage [16]. In a recent in vitro study, we mimicked these effects of neuroinflammation in vivo by activating microglia with endotoxin and studied the effects of microglia-conditioned medium on the Nrf2 system in astrocytes after 24 and $72 \mathrm{~h}$ of treatment. These studies showed that inflammation may have dual, time-related, effects on the Nrf2 system that were dependent on protein kinase activation, i.e. activation of Akt, c-Jun N-terminal kinase (JNK) and extracellular regulated kinase (ERK) stimulated the Nrf2 system, whereas sustained activation of glycogen synthase kinase-3 $\beta$ (GSK3 $\beta$ ) and p38 down-regulated the system [17]. Another factor that has complementary effects on the anti-oxidant system is the peroxisome proliferator-activated receptor- $\gamma$ coactivator $1 \alpha$ (PGC- $1 \alpha$ ), which functions as a cofactor for many transcription factors [18]. Activation of PGC-1 1 results in elevated levels of manganese superoxide dismutase (MnSOD) present in the mitochon- dria. Earlier, it has been shown that LPS treatment increases MnSOD, both in vitro and in vivo $[19,20]$.

Here, we have employed the in vivo model and investigated how inflammation by systemic LPS $(0.3 \mathrm{mg} / \mathrm{kg}$ of LPS, i.p.) affects the Nrf2 and PGC-1 $1 \alpha$ systems in the brain after 24 and $72 \mathrm{~h}$.

\section{Materials and Methods}

\section{Animal Treatment and Tissue Preparation}

Sprague-Dawley rats from our in-house colony were housed in a 12-hour light-dark cycle and bred at Experimental Biomedicine (Gothenburg University, Gothenburg, Sweden) and were provided with a standard laboratory chow diet (B\&K, Solna, Sweden) and drinking water ad libitum. All animal experiments were approved by the Ethical Committee of Gothenburg (No. 264-2009) and followed the guidelines for the care and use of experimental animals and the European Communities Council Directive of 24 November 1986 (86/609/EEC).

Rats were injected intraperitoneally with saline or LPS $(0.3 \mathrm{mg} /$ $\mathrm{kg}$ ) at post-natal day 7 , and brain tissue was collected at $24 \mathrm{~h}$ (saline group, $\mathrm{n}=9$; LPS group, $\mathrm{n}=11$ ) and $72 \mathrm{~h}$ (saline group, $\mathrm{n}=9$; LPS group, $n=11$ ) after LPS exposure for Western blot assessment. Animals were deeply anaesthetized and perfused intracardially with ice-cold saline. Brains were rapidly removed and frozen in liquid nitrogen and stored at $-80^{\circ} \mathrm{C}$ until further use.

After thawing, ice-cold isolation buffer (50 mM Tris, $\mathrm{pH} 7.3$; 2 mM EDTA, $1 \%$ protease inhibitor cocktail) was added and homogenization was performed gently with a manual homogenizer (VWR International). The cytosolic and nuclear fractions were separated according to a method described previously [33]. Briefly, the homogenates were centrifuged at $800 \mathrm{~g}$ for $10 \mathrm{~min}$ at $4^{\circ} \mathrm{C}$. The pellets were washed in homogenizing buffer and recentrifuged, producing a crude nuclear pellet (P1). The supernatant was further centrifuged at $9,200 \mathrm{~g}$ for $15 \mathrm{~min}$ at $4^{\circ} \mathrm{C}$, producing a crude mitochondrial fraction in the pellet (P2) and a cytosolic fraction in the supernatant. All fractions were stored at $-80^{\circ} \mathrm{C}$. P1 and supernatant fractions were used for immunoblotting.

\section{Western Blot Analysis}

Homogenate fractions were mixed with $5 \times$ Laemmle sample buffer and boiled for $5 \mathrm{~min}$. Then, an equal amount of protein (30 $\mu \mathrm{g}$ ) was resolved on $10 \%$ SDS-PAGE in a MOPS or MES buffer (Invitrogen, Carlsbad, Calif., USA) and electroblotted at $40 \mathrm{~V}$ for $70 \mathrm{~min}$ at $4^{\circ} \mathrm{C}$ to nitrocellulose (Bio-Rad, Hercules, Calif., USA). The membranes were blocked for $1 \mathrm{~h}$ at room temperature in $5 \%$ (w/v) dry skimmed milk (Semper Mjölk, Sundyberg, Sweden) in TBS with $0.1 \%$ Tween 20 (TBST). Then, the membranes were incubated overnight at $4{ }^{\circ} \mathrm{C}$ with the corresponding primary antibodies in 5\% bovine serum albumin-TBST, extensively washed with TBST solution and incubated with the correspondent secondary antibodies for $1 \mathrm{~h}$ at room temperature. Finally, the blots were rinsed and the peroxidase reaction was developed by enhanced chemiluminescence SuperSignal ${ }^{\circledR}$ West Dura Extended Duration Substrate (Thermo Scientific, Rockford, Ill., USA). Blots were stripped in Restore ${ }^{\mathrm{TM}}$ Plus Western Blot Stripping Buffer (Thermo Scientific) and were re-probed sequentially. 


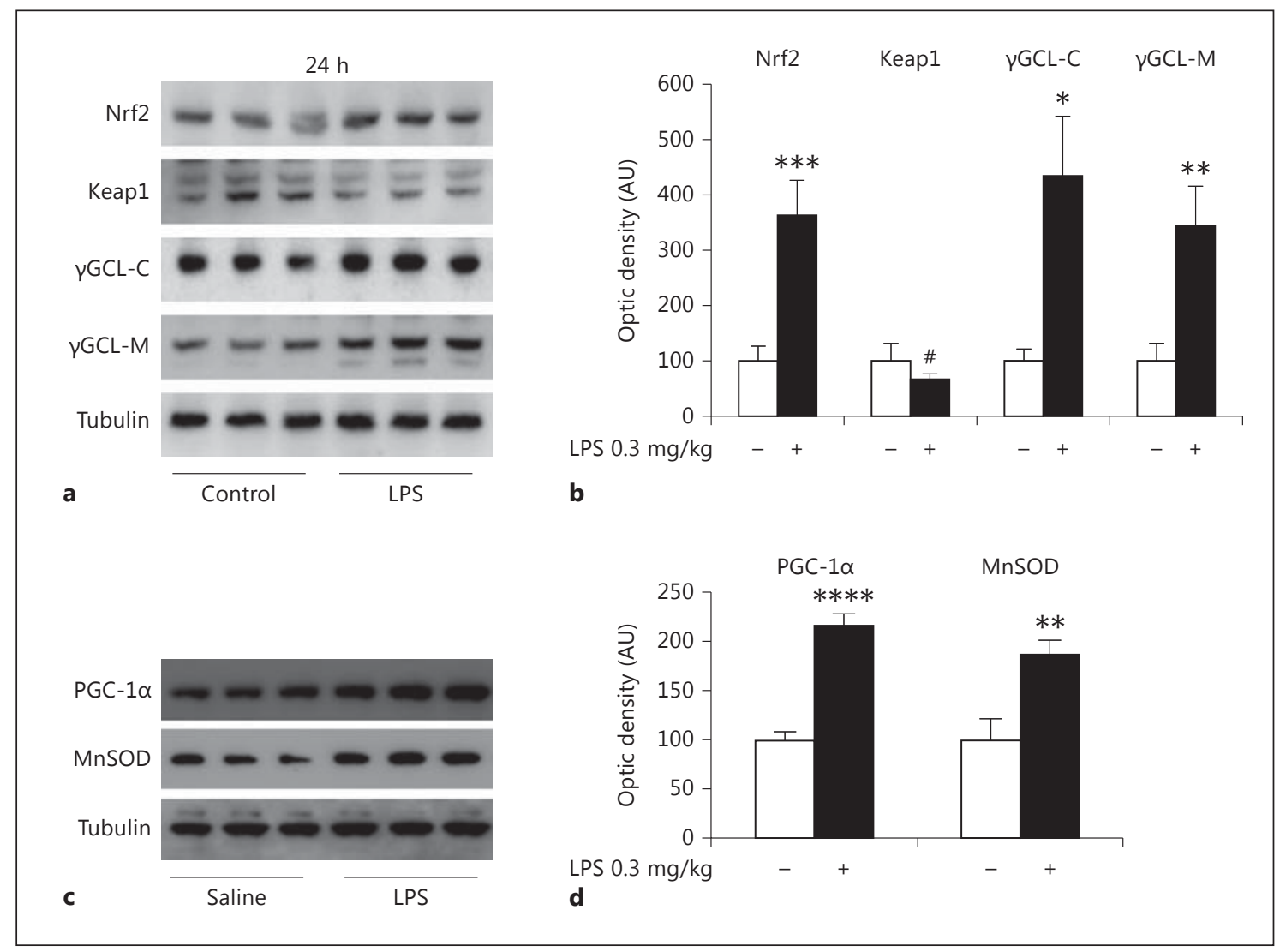

Fig. 1. Systemic LPS ( $0.3 \mathrm{mg} / \mathrm{kg}$, i.p.) induced the antioxidant defence systems after $24 \mathrm{~h}$. a Seven-day-old rat pups were exposed to either saline $(\mathrm{n}=9)$ or LPS $(0.3 \mathrm{mg} / \mathrm{kg} ; \mathrm{n}=11)$ for $24 \mathrm{~h}$ after which their brains were removed for the protein expression analysis of Nrf2, Keap1, $\gamma$ GCL-C and $\gamma$ GCL-M subunits. b Densitometric analysis of Western blot. ${ }^{*} \mathrm{p}<0.05$ versus saline; ${ }^{* *} \mathrm{p}<0.01$ versus saline; ${ }^{* * *} \mathrm{p}<0.005$ versus saline; ${ }^{*} \mathrm{p}<0.05$ versus saline (MannWhitney U test). c A similar analysis was performed for the protein expression of PGC-1 $\alpha$ and MnSOD. d Densitometric analysis. ${ }_{* * * *} \mathrm{p}<0.001$ versus saline, ${ }^{* *} \mathrm{p}<0.01$ versus saline. Results are expressed as the mean \pm SEM. AU $=$ Arbitrary units.
Images were captured with a Fujifilm Image Reader LAS-1000 Pro version 2.6 (Stockholm, Sweden), and the different band intensities (density arbitrary units) corresponding to immunoblot detection of protein samples were quantified using the Fujifilm Multi Gauge version 3.0 software (Stockholm, Sweden).

\section{Antibodies}

Anti-histone $\mathrm{H} 2 \mathrm{~B}$, anti-acetyl-histone $\mathrm{H} 3$, anti-acetyl-histone $\mathrm{H} 4$ and anti-trimethyl-Lys9-histone H3 were from Millipore (Solna, Sweden). Anti-phosphorylated p38 and anti-phosphorylated Akt were from New England Biolabs (Beverly, Mass., USA). AntiNrf2 was from R\&D Diagnostics (Minneapolis, Minn., USA). Anti-Keap1, anti-PGC-1 $\alpha$, anti-actin, anti- $\alpha$-tubulin, anti- $\gamma$ glutamylcysteine ligase catalytic subunit ( $\gamma$ GCL-C) and anti- $\gamma$ glutamylcysteine ligase modulatory subunit ( $\gamma$ GCL-M) antibodies were from Santa Cruz Biotechnology (Heidelberg, Germany). Anti-MnSOD was from Lab Frontier (Seoul, South Korea). Peroxidase-conjugated anti-rabbit and anti-mouse secondary antibodies were from Vector Laboratories (Burlingame, Calif., USA).

\section{Statistical Analysis}

Results are presented as means \pm standard error of the mean (SEM). Student's t test was used to determine statistical significance $(95 \% ; p<0.05)$. If Levene's test for homogeneity of variances was significant, then the non-parametric Mann-Whitney U test was used.

\section{Results}

\section{Effect of 24-Hour Systemic LPS Exposure on the} Cerebral Nrf2 System

In our earlier studies, we showed that conditioned medium from LPS-activated microglia can have positive effects after $24 \mathrm{~h}$ but negative effects after $72 \mathrm{~h}$ on the Nrf2 system in astrocyte-rich cultures [21]. Similarly, dual effects of LPS with protection after $24 \mathrm{~h}$ and sensitization 


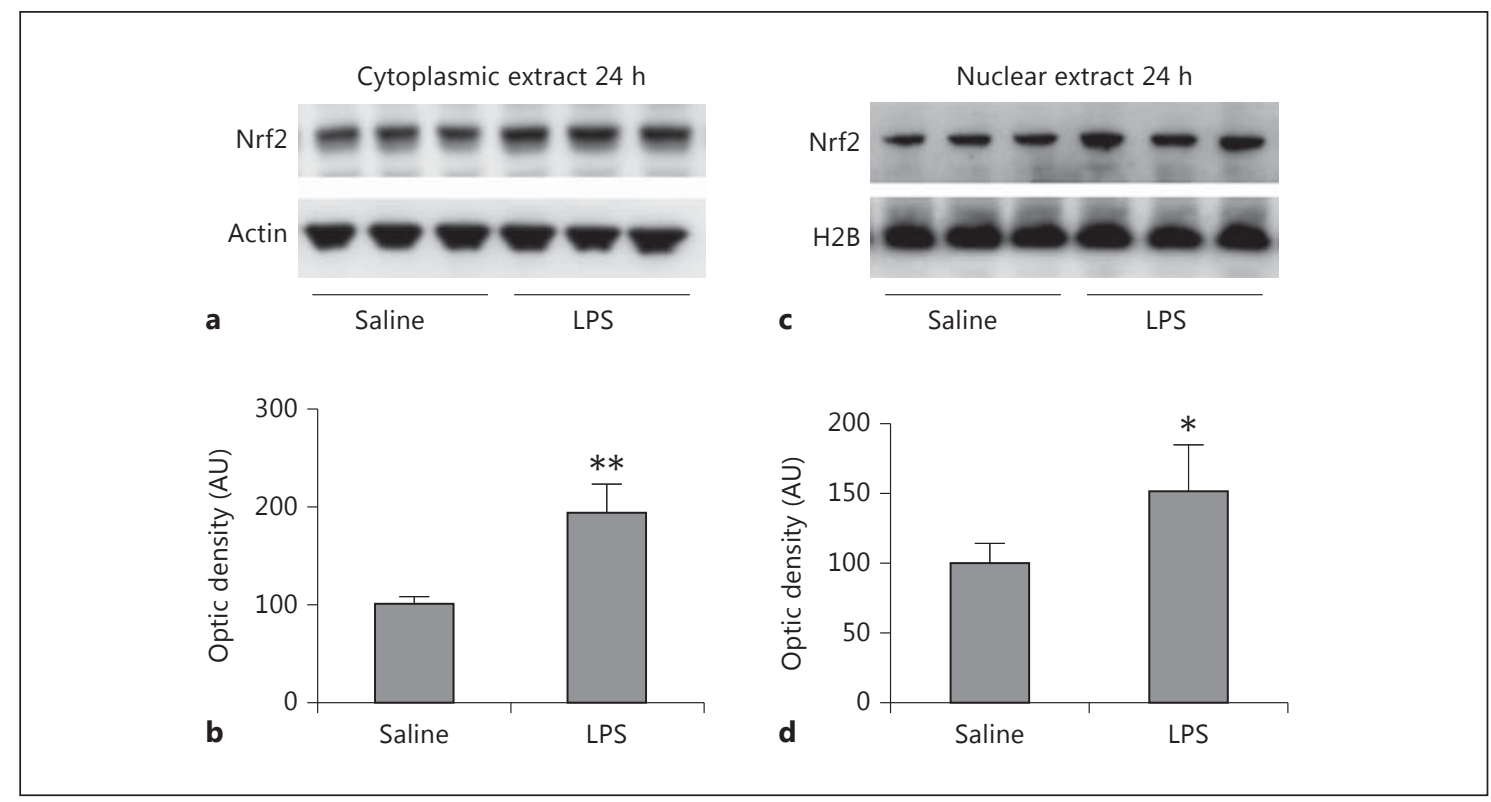

Fig. 2. Nuclear and cytosolic extracts from brain homogenates of rats injected with LPS $(0.3 \mathrm{mg} / \mathrm{kg}$, i.p.) showed increased $\mathrm{Nrf} 2$ protein levels after $24 \mathrm{~h}$. a Nrf2 protein level in cytoplasmic extract of brains of 7-day-old rats $24 \mathrm{~h}$ after injection (i.p.) of either saline or LPS $(0.3 \mathrm{~g} / \mathrm{kg})$. b Densitometric analysis of the Western blots.
** $\mathrm{p}<0.01$ versus saline. $\mathrm{c}$ A similar analysis was performed for the protein expression of $\mathrm{Nrf} 2$ in the nuclear fraction. $\mathbf{d}$ Densitometric analysis. ${ }^{*} \mathrm{p}<0.05$ versus saline. Results are expressed as the mean \pm SEM. AU $=$ Arbitrary units.

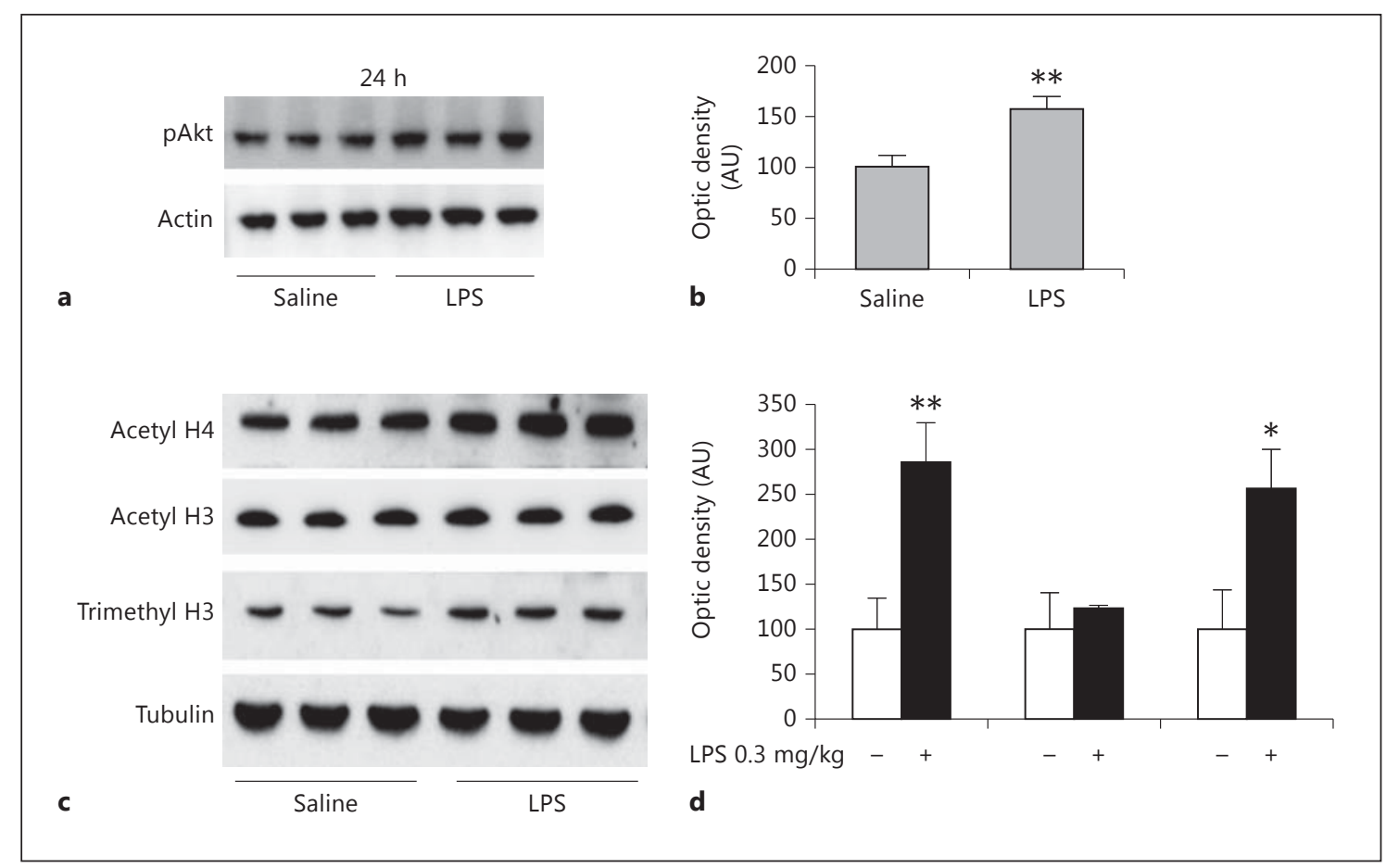

Fig. 3. a Systemic LPS ( $0.3 \mathrm{mg} / \mathrm{kg}$, i.p. $)$ increased the level of phosphorylated Akt (pAkt) after 24 h. b Densitometric analysis of Western blot. ${ }^{* *} \mathrm{p}<0.01$ versus saline. c Exposure for $24 \mathrm{~h}$ to LPS $(0.3 \mathrm{mg} / \mathrm{kg}$, i.p.) modified the acetylation/methylation pattern of histones $\mathrm{H} 4$ and H3. d Densitometric analysis. ${ }^{*} \mathrm{p}<0.05$ versus saline; ${ }^{* *} \mathrm{p}<0.01$ versus saline. Results are expressed as the mean \pm SEM. AU $=$ Arbitrary units. 


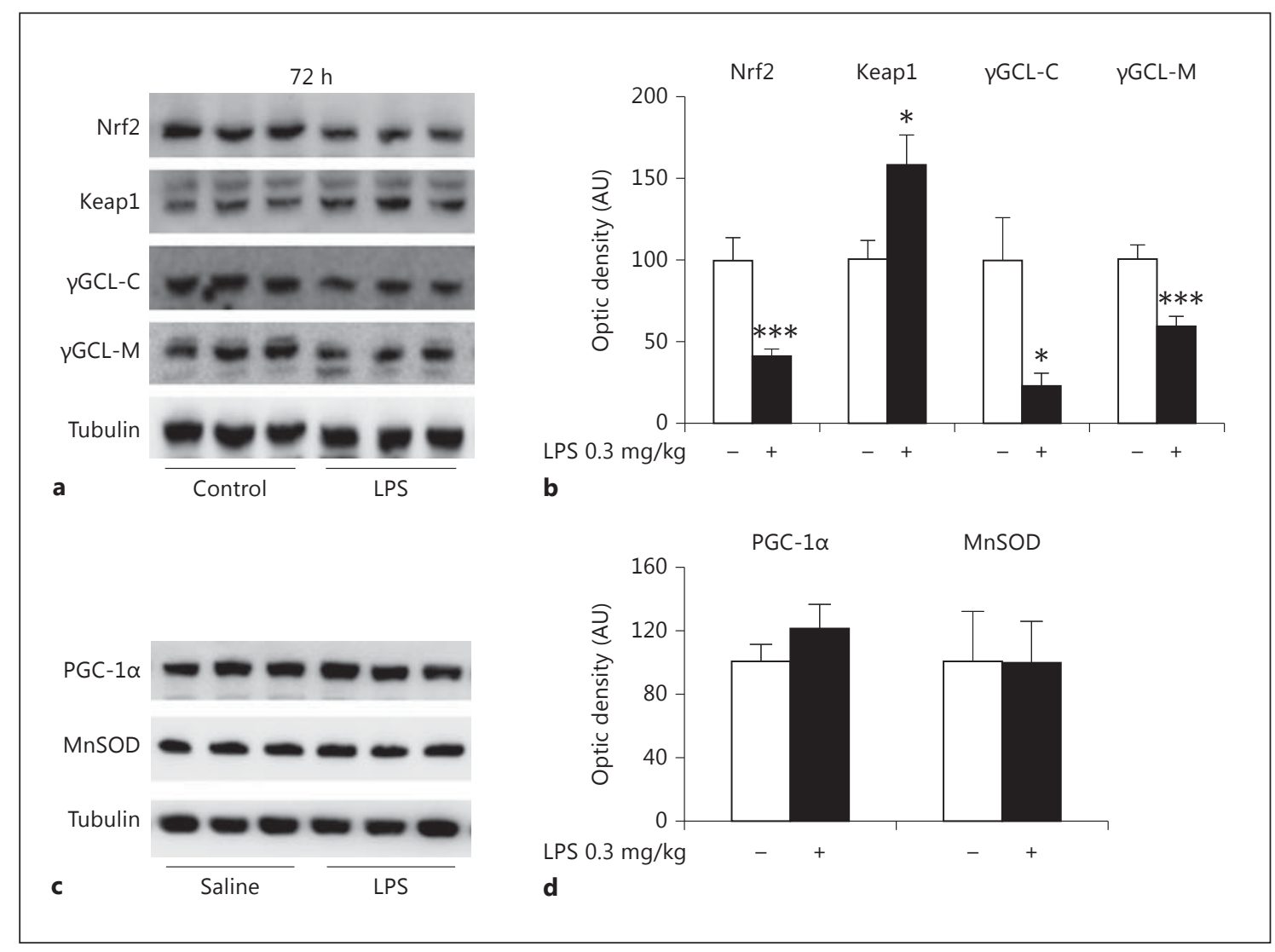

Fig. 4. Systemic LPS ( $0.3 \mathrm{mg} / \mathrm{kg}$, i.p. $)$ caused decreased antioxidant defence levels after $72 \mathrm{~h}$. a Seven-day-old rat pups were injected with saline $(\mathrm{n}=11)$ or LPS $(0.3 \mathrm{mg} / \mathrm{kg} ; \mathrm{n}=9)$ and, after $72 \mathrm{~h}$, their brains were removed for protein level analysis of Nrf2, Keap1, $\gamma$ GCL-C and $\gamma$ GCL-M subunits. b Densitometric analysis of West-

ern blot. ${ }^{*} \mathrm{p}<0.05$ versus saline; ${ }^{* * *} \mathrm{p}<0.005$ versus saline. c A similar analysis was performed for the protein expression of PGC$1 \alpha$ and MnSOD. No changes were observed in these protein levels. d Densitometric analysis. Results are expressed as the mean \pm SEM. AU = Arbitrary units.

after $72 \mathrm{~h}$ on a hypoxic-ischaemic insult were demonstrated in neonatal rats [15]. Therefore, we evaluated the effects of systemic LPS after 24 and $72 \mathrm{~h}$ on the Nrf2 system in vivo in this study.

The effects of 24-hour exposure of 7-day-old rats to 0.3 $\mathrm{mg} / \mathrm{kg}$ LPS (i.p.) on the components of the brain-inducible Nrf2 and PGC-1 $\alpha$ systems are illustrated in figure 1. We first analysed the effects of LPS on cerebral Nrf2, Keap1, $\gamma$ GCL-C and $\gamma$ GCL-M protein expression (fig. 1a). As shown in figure 1b, the densitometric analysis of Western blot showed that 24-hour systemic LPS induced an increased expression of Nrf2, $\gamma$ GCL-C and $\gamma$ GCL-M but reduced the level of Keap1. Similarly, $24 \mathrm{~h}$ after LPS exposure, there was an increase in the protein levels of PGC$1 \alpha$ and MnSOD (fig. 1c), as shown in the densitometric analysis (fig. 1d).

Systemic LPS Modulates Antioxidant Defence in the Brain
Moreover, we analysed the cytoplasmic and nuclear fractions of the brain homogenates and found that there was an increase in Nrf2 protein levels in both fractions (fig. 2).

To investigate the possible mechanisms relevant to the Nrf2-related changes observed above, we further analysed changes of the mitogen-activated protein kinase (MAPK) signal pathway and cerebral acetylation and methylation patterns of histones. The analysis of the MAPK signalling pathways activated in the brain by the systemic administration of LPS ( $24 \mathrm{~h}$ ) showed increased phosphorylation levels of Akt (fig. 3a). The levels of phosphorylated p38 MAPK and JNK were below detection limits. The systemic treatment with LPS for $24 \mathrm{~h}$ also induced changes in the cerebral acetylation and methylation of histones. Thus, an increase in the acetylation of histone $\mathrm{H} 4$ and in the methylation of histone $\mathrm{H} 3-\mathrm{K} 9$ was

Neuroimmunomodulation 2013;20:185-193 


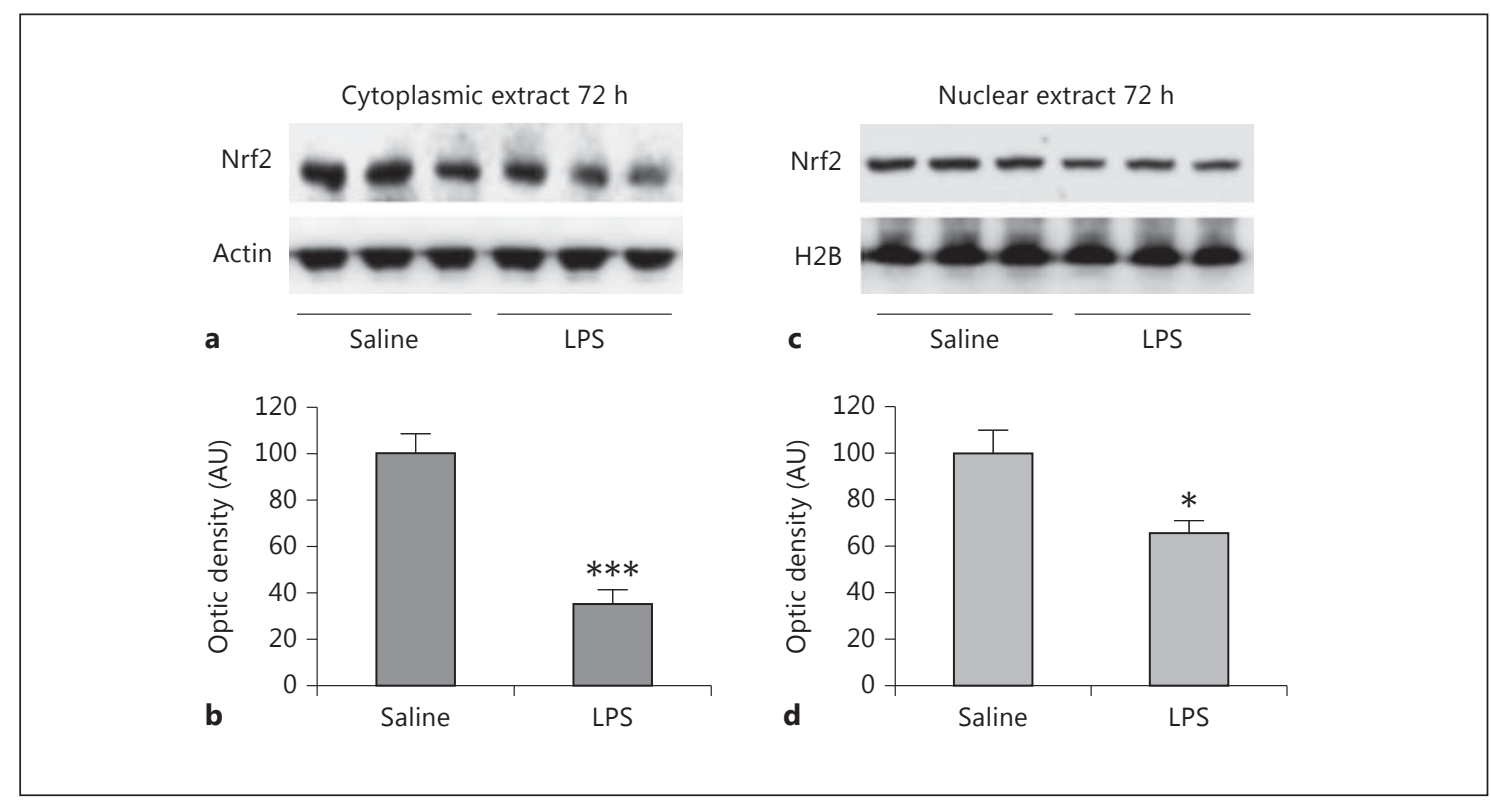

Fig. 5. Nuclear and cytosolic extracts from brain homogenates of rats injected with LPS $(0.3 \mathrm{mg} / \mathrm{kg}$, i.p.) showed decreased levels of Nrf2 protein levels after $72 \mathrm{~h}$. a The levels of Nrf2 protein in the cytosolic fraction of brain extracts $72 \mathrm{~h}$ after injection of saline or LPS $(0.3 \mathrm{mg} / \mathrm{kg})$ in 7 -day-old rats. b Densitometric analysis of
Western blot. ${ }^{* * *} \mathrm{p}<0.005$ versus saline. c A similar analysis was performed for the protein expression of Nrf2 in the nuclear fraction. $\mathbf{d}$ Densitometric analysis. ${ }^{*} \mathrm{p}<0.05$ versus saline. Results are expressed as the mean \pm SEM. AU $=$ Arbitrary units. detected with no changes in the acetylation pattern of $\mathrm{H} 3$ (fig. 3c, d).

\section{Effect of 72-Hour Systemic LPS on the Cerebral Nrf2 System}

The effects of 72-hour exposure to $0.3 \mathrm{mg} / \mathrm{kg}$ LPS (i.p.) on the components of the brain-inducible Nrf2 and PGC-1a systems in 7-day-old rats are shown in figure 4. We first analysed the effects of LPS on cerebral Nrf2, Keap1, $\gamma$ GCL-C and $\gamma$ GCL-M protein expression (fig. 4a). The densitometric analysis of Western blot bands showed that $72 \mathrm{~h}$ after systemic LPS injection, expression of Nrf2, $\gamma$ GCL-C and $\gamma$ GCL-M was lower, but higher levels of Keap1 were observed when compared to saline treatment (fig. 4b). Next, the protein levels of PGC-1 $\alpha$ and MnSOD were analysed (fig. 4c); however, no changes were observed, as shown in the densitometric analysis (fig. $4 \mathrm{~d}$ ).

When the cytoplasmic and nuclear fractions were analysed, we found that in both fractions the protein levels of Nrf2 were reduced compared to saline control (fig. 5).

The analysis of the signalling pathways activated in the brain by the systemic administration of LPS ( $72 \mathrm{~h}$ ) showed unchanged phosphorylation levels of Akt with a concom- itant increase in the phosphorylation levels of p38 MAPK (fig. 6a, b). Both JNK and ERK1/2 were below detection limits (data not shown). Interestingly, $72 \mathrm{~h}$ following the systemic treatment with LPS, there were no changes in the cerebral acetylation and methylation pattern of histones, albeit a tendency to lower acetylation levels of histone $\mathrm{H} 3$ which did not reach statistical significance (fig. 6c, d).

\section{Discussion}

LPS elevated the protein levels of Nrf2 and its downstream products, $\gamma \mathrm{GCL}-\mathrm{M}$ and $\gamma \mathrm{GCL}-\mathrm{C}, 24 \mathrm{~h}$ after systemic administration. This is in agreement with our earlier finding that systemic LPS in neonatal rats increased brain levels of Nrf2 mRNA after $24 \mathrm{~h}$ [22]. In addition, the complementary transcriptional cofactor PGC-1 $\alpha$ and MnSOD, whose transcription is elevated by PGC- $1 \alpha$, were raised at this time point. These effects point towards an induced anti-oxidant defence by LPS $24 \mathrm{~h}$ after treatment. Such elevation in the anti-oxidant systems may well underlie our previously observed preconditioning effect and the protection by LPS in neonatal rats $24 \mathrm{~h}$ after 


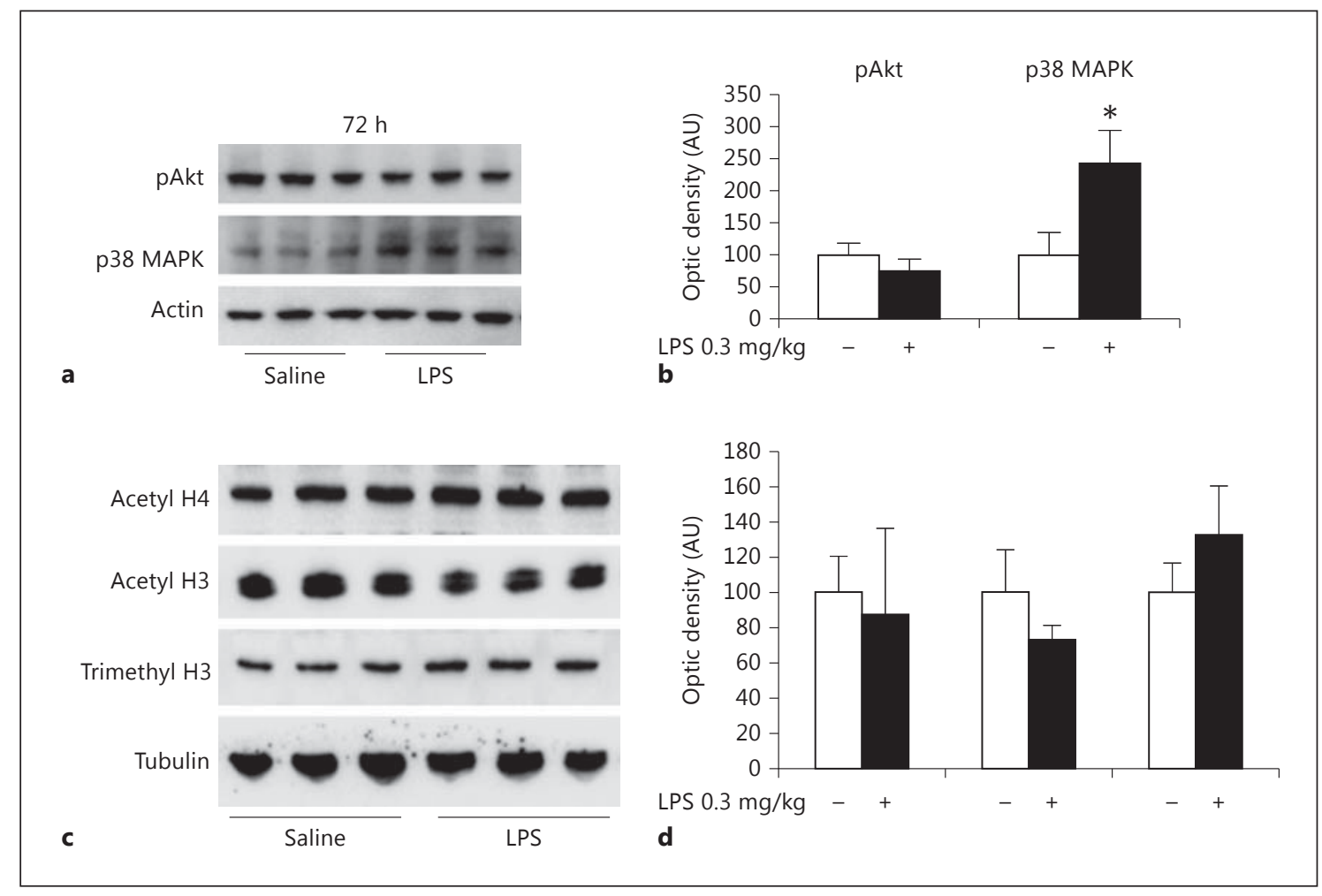

Fig. 6. a Systemic LPS $(0.3 \mathrm{mg} / \mathrm{kg}$, i.p. $)$ activated p38 MAPK. b Densitometric analysis of Western blot. ${ }^{*} \mathrm{p}<0.05$ versus saline. c The acetylation pattern of histone $\mathrm{H} 3$ showed a tendency (al- though without statistical significance) to be down-regulated $72 \mathrm{~h}$ following systemic LPS $(0.3 \mathrm{mg} / \mathrm{kg}$, i.p.). d Densitometric analysis. Results are expressed as the mean \pm SEM. AU = Arbitrary units. administration [16]. This also agrees well with the induction of the Nrf2 system $24 \mathrm{~h}$ after LPS exposure that we observed with microglia-conditioned medium in primary astrocytes [21].

The mechanism behind the increase in the Nrf2 system $24 \mathrm{~h}$ after LPS administration could be related to activation of certain kinases. We observed previously that inhibition of ERK1/2, Akt and JNK reduced Nrf2-mediated transcription in astrocytes $[17,21]$. This is in accord with a recent report showing that LPS-stimulated expression of heme oxygenase 1 , a down-stream product of Nrf2 transcription, involved Akt and ERK1/2 activation [23]. In the present study, the levels of phosphorylated/activated Akt were increased $24 \mathrm{~h}$ after LPS exposure (JNK was below detection limit and ERK1/2 too variable). It is thus possible that the observed protection against hypoxic-ischaemic injury $24 \mathrm{~h}$ after LPS treatment is partly mediated via activation of Akt, in accordance with earlier studies on hypoxic pre-conditioning in vivo [24]. In parallel with the activation of the Nrf2 system $24 \mathrm{~h}$ after LPS exposure, we observed a profound down-regulation of the sequester protein Keap1. Similar down-regulation of Keap1 and up-regulation of Nrf2 has also been observed in the brain after MCA occlusion in adult mice [12]. The reason for the opposite effects on Nrf2 and Keap1 levels might be that activation of Nrf2 shifts the ubiqutination (and degradation) from Nrf2 to Keap1 [25].

One additional mechanism that may be involved in the observed positive effects on the Nrf2 system is the acetylation of proteins, i.e. histones, transcription factors or both. We recently showed that down-regulation of the $\mathrm{Nrf} 2$ system by medium from LPS $(10 \mathrm{ng} / \mathrm{ml})$ activated microglia was parallel to decreased acetylation of $\mathrm{H} 3$ and elevated trimethylation of H3-K9. These effects of microglia-conditioned medium on both the Nrf2 system and the histone acetylation levels were reversed by histone deacetylase (HDAC) inhibitors [17]. Here, activation of the Nrf2 system was accompanied by elevated acetylation of $\mathrm{H} 4$ and increased trimethylation of $\mathrm{H} 3$. On the basis of a study showing that tumour necrosis factor (TNF)- $\alpha$ and $\mathrm{H}_{2} \mathrm{O}_{2}$ elevate $\mathrm{H} 4$ histone acetylation in vitro [26], we hypothesize that the elevated acetylation level of 
$\mathrm{H} 4$ in vivo may be related to TNF- $\alpha$ or oxidative stress induced by systemic LPS treatment.

The levels of H3-K9 trimethylation was elevated $24 \mathrm{~h}$ after systemic LPS but restored to control levels after $72 \mathrm{~h}$. Trimethylation of $\mathrm{H} 3-\mathrm{K} 9$ is generally associated with repression of transcription and dimethylation of $\mathrm{H} 3-\mathrm{K} 9$ with binding of DNA methylases that can cause more persistent epigenetic modification of $\mathrm{CpG}$ islands in various promoters [27]. These effects of $\mathrm{H} 3-\mathrm{K} 9$ may thus underlie the down-regulation of genes in the rat brain after systemic administration of LPS [16]. However, it should be noted that $\mathrm{H} 3-\mathrm{K} 9$ trimethylation in transcribed regions has been correlated with active gene transcription rather than with transcriptional repression [28]. Obviously, to confirm the potential significance of the H3-K9 trimethylation on Nrf2/PGC-1a-mediated transcription, further more detailed studies are needed.

The increased acetylation levels of $\mathrm{H} 4$ imply elevated activity of histone acetyl transferases (HATs), which implicate that also Nrf2 is acetylated. Elevated acetylation of Nrf2 by HATs such as CBP increases transcription mediated by Nrf2 which could be one mechanism behind the up-regulation of the Nrf2 system observed in our study [29]. The apparent elevated HAT activity could be related to the increase in Akt activation as nuclear Akt interacts with and phosphorylates $\mathrm{p} 300$ in response to TNF- $\alpha$ [30].

Up-regulation of MnSOD after LPS treatment in adult rats has been observed earlier in in vivo and in vitro studies [20]. In that study, LPS administration reduced the infarct area when given $72 \mathrm{~h}$ prior to MCA occlusion [20]. The levels of MnSOD were measured 12 and $72 \mathrm{~h}$ following LPS but were only increased after $72 \mathrm{~h}$. In our case, we observed a significant increase in MnSOD after $24 \mathrm{~h}$ but no increase at $72 \mathrm{~h}$ after LPS. The apparent discrepancy between the results most certainly relates to the differences in models, i.e., we used 7-day-old rats and $0.3 \mathrm{mg}$ LPS $/ \mathrm{kg}$, whereas Bordet and co-workers [20] employed adult rats and used $0.5 \mathrm{mg}$ LPS $/ \mathrm{kg}$. TNF- $\alpha$ was suggested to mediate the protective effects of LPS treatment and the increase in MnSOD.

After $72 \mathrm{~h}$, the effect of systemic LPS administration on the Nrf2 system was more or less reversed in the present study, i.e., Nrf2, $\gamma$ GCL-M and $\gamma$ GCL-C levels were down-regulated, whereas Keap1 levels were higher compared to saline-treated controls. This is in most respects similar to our results in vitro for the effects of microgliaconditioned medium on the Nrf2 system in astrocyte cultures $[17,21]$.

We have earlier evaluated the role of kinases in the upand down-regulation of the Nrf2 system [17, 21]. These in vitro studies showed that microglia-conditioned medium exerts negative effects on the Nrf2 system via p38 MAPK and GSK3 $\beta$ activation, whereas positive effects were observed for activation of JNK, ERK and Akt/PI3 kinase. Here, we analysed activation of p38 and Akt (phosphorylated GSK3 $\beta$ and JNK were below detection limit and ERK1/2 showed too high a variation). The level of phosphorylated p38 MAPK was up-regulated at $72 \mathrm{~h}$ after LPS administration, in accordance with our previous in vitro studies showing that when the Nrf2 system was down-regulated, p38 MAPK was phosphorylated. The elevated level of p38 MAPK can probably explain the decreased level of Nrf2 in the nucleus. Genetic deletion and pharmacological inhibition of p38 MAPK have earlier been shown to increase export of p 38 MAPK from the nucleus and to decrease Nrf2-mediated transcription of phase II genes [31, 32].

As noted above, we have shown that medium from 72hour LPS (10 $\mathrm{ng} / \mathrm{ml})$ activated microglia down-regulated the Nrf2 system in astrocytes, which occurred in parallel to decreased acetylation of $\mathrm{H} 3, \mathrm{H} 4$, elevated activity of HDACs and increased trimethylation of H3-K9. The effects on both the Nrf2 system and the histone acetylation levels were reversed by HDAC inhibitors, which also protected against oxidative stress-induced cell damage. Interestingly, the deacetylation was obliterated by inhibitors of p38 MAPK and GSK3 $\beta$ [17]. Here, we did not observe any significant changes in $\mathrm{H} 3$ acetylation after $72 \mathrm{~h}$, although a trend towards decreased acetylation was observed. The data show clearly that between 24 and $72 \mathrm{~h}$, the acetylation level of $\mathrm{H} 4$ is decreased, meaning that either the $\mathrm{H} 4$ acetylation by HATs is decreased or that the high acetylation level at $24 \mathrm{~h}$ is decreased by elevated HDAC activity. This fits well with our in vitro results where we showed that after $72 \mathrm{~h}$ but not after $24 \mathrm{~h}$ microglia-conditioned medium caused elevated HDAC activity in astrocytes [17].

In summary, systemic LPS in neonatal rats induced time-dependent changes in Nrf2 and PGC-1 $\alpha$ that correlated well with the protective effect after $24 \mathrm{~h}$ and the deleterious effects after $72 \mathrm{~h}$ of systemic LPS reported earlier $[15,16]$. Thus, up-regulations of Nrf2, the modulatory and catalytic subunits of $\gamma \mathrm{GCL}$, as well as PGC- $1 \alpha$ and MnSOD were observed at $24 \mathrm{~h}$ after LPS treatment whereas Nrf2 and $\gamma \mathrm{GCL}$ subunits were down-regulated and PGC- $1 \alpha$ and MnSOD were normalized at $72 \mathrm{~h}$ following LPS treatment. The positive effects on the inducible antioxidant defence after $24 \mathrm{~h}$ correlated with activated Akt and increased acetylation of $\mathrm{H} 3$, whereas the negative effect after $72 \mathrm{~h}$ was in parallel with elevated p38 MAPK activation and normalized Akt and $\mathrm{H} 3$ acetylation. 


\section{Acknowledgments}

The expert technical assistance of Barbro Jilderos and Mariette Berlin is gratefully acknowledged. The work was supported by the Swedish Research Council/Medicine and Åhlén-stiftelsen. M.S. is supported by the National Institutes of Health (GM 44842). C.M. is supported by VR (K2007-54x-14185-06-3), FP6, Neobrain (036534), the Foundation Leducq and the European Union (HEALTH-F2-2009-241778 421). X.W. is supported by the Swedish Medical Research Council (VR K2009-54X-21119-01-4) and the Bill and Melinda Gates Foundation Grand Challenge (OPP1036135).

\section{References}

1 Coyle JT, Puttfarcken P: Oxidative stress, glutamate, and neurodegenerative disorders. Science 1993;262:689-695.

-2 Frank-Cannon TC, Alto LT, McAlpine FE, Tansey MG: Does neuroinflammation fan the flame in neurodegenerative diseases? Mol Neurodegener 2009;4:47.

-3 Innamorato NG, Rojo AI, García-Yagüe AJ, Yamamoto M, de Ceballos ML, Cuadrado A: The transcription factor Nrf2 is a therapeutic target against brain inflammation. J Immunol 2008; 181:680-689.

4 Chan K, Xiao-Dong H, Yuet Wai K: An important function of Nrf2 in combating oxidative stress: detoxification of acetaminophen. Proc Natl Acad Sci USA 2001;98: 4611-4616.

5 Hubbs AF, Benkovic SA, Miller DB, O'Callaghan JP, Battelli L, Schwegler-Berry D, Ma Q: Vacuolar leukoencephalopathy with widespread astrogliosis in mice lacking transcription factor Nrf2. Am J Pathol 2007;170:20682076.

6 Zhao Z, Chen Y, Wang J, Sternberg P, Freeman ML, Grossniklaus HE, Cai J: Age-related retinopathy in NRF2-deficient mice. PLoS One 2011;6:e19456.

-7 Ramsey CP, Glass CA, Montgomery MB, Lindl KA, Ritson GP, Chia LA, Hamilton RL, Chu CT, Jordan-Sciutto KL: Expression of Nrf2 in neurodegenerative diseases. J Neuropathol Exp Neurol 2007;66:75-85.

8 Shih AY, Li P, Murphy TH: A small-molecule-inducible Nrf2-mediated antioxidant response provides effective prophylaxis against cerebral ischemia in vivo. J Neurosci 2005;25: 10321-10335.

9 Zhao J, Kobori N, Aronowski J, Dash PK: Sulforaphane reduces infarct volume following focal cerebral ischemia in rodents. Neurosci Lett 2006;393:108-112.

10 Yang C, Zhang X, Fan H, Liu Y: Curcumin upregulates transcription factor $\mathrm{Nrf} 2, \mathrm{HO}-1$ expression and protects rat brains against focal ischemia. Brain Res 2009;1282:133-141.

11 Shah ZA, Li RC, Ahmad AS, Kensler TW, Yamamoto M, Biswal S, Doré S: The flavanol (-)-epicatechin prevents stroke damage through the Nrf2/HO1 pathway. J Cereb Blood Flow Metab 2010;30:1951-1961.

12 Tanaka N, Ikeda Y, Ohta Y, Deguchi K, Tian F, Shang J, Matsuura T, Abe K: Expression of Keap1-Nrf2 system and antioxidative proteins in mouse brain after transient middle cerebral artery occlusion. Brain Res 2011;1370: 246-253.
13 Ping Z, Liu W, Kang Z, Cai J, Wang Q, Cheng N, Wang S, Wang S, Zhang JH, Sun X: Sulforaphane protects brains against hypoxicischemic injury through induction of Nrf2dependent phase 2 enzyme. Brain Res 2010 1343:178-185.

14 Wallin C, Puka-Sundvall M, Hagberg H, Weber SG, Sandberg M: Alterations in glutathione and amino acid concentrations after hypoxia-ischemia in the immature rat brain. Brain Res Dev Brain Res 2000;125:51-60.

15 Wang X, Svedin P, Nie C, Lapatto R, Zhu C, Gustavsson M, Sandberg M, Karlsson JO, Romero R, Hagberg H, Mallard C: N-acetylcysteine reduces lipopolysaccharide-sensitized hypoxic-ischemic brain injury. Ann Neurol 2007;61:263-271

16 Eklind S, Mallard C, Arvidsson P, Hagberg H: Lipopolysaccharide induces both a primary and a secondary phase of sensitization in the developing rat brain. Pediatr Res 2005;58: 112-116.

17 Correa F, Mallard C, Nilsson M, Sandberg M: Activated microglia decrease histone acetylation and Nrf2-inducible anti-oxidant defence in astrocytes: restoring effects of inhibitors of HDACs, p38 MAPK and GSK3beta. Neurobiol Dis 2011;44:142-151.

18 Clark J, Simon DK: Transcribe to survive: transcriptional control of antioxidant defence programs for neuroprotection in Parkinson's disease. Antioxid Redox Signal 2009;11:509-528.

19 Kifle Y, Monnier J, Chesrown SE, Raizada MK, Nick HS: Regulation of the manganese superoxide dismutase and inducible nitric oxide synthase gene in rat neuronal and glial cells. J Neurochem 1996;66:2128-2135.

20 Bordet R, Deplanque D, Maboudou P, Puisieux F, Pu Q, Robin E, Martin A, Bastide M, Leys D, Lhermitte M, Dupuis B: Increase in endogenous brain superoxide dismutase as a potential mechanism of lipopolysaccharideinduced brain ischemic tolerance. J Cereb Blood Flow Metab 2000;20:1190-1196.

-21 Correa F, Ljunggren E, Mallard C, Nilsson M, Weber SG, Sandberg M: The Nrf2-inducible antioxidant defense in astrocytes can be both up- and down-regulated by activated microglia: involvement of p38 MAPK. Glia 2011; 59:785-799.

22 Mallard C, Hagberg H: Inflammation-induced preconditioning in the immature brain. Semin Fetal Neonatal Med 2007;12: 280-286.

23 Park PH, Hur J, Kim YC, An RB, Sohn DH: Involvement of heme oxygenase-1 induction in inhibitory effect of ethyl gallate isolated from Galla Rhois on nitric oxide production in RAW 264.7 macrophages. Arch Pharm Res 2011;34:1545-1552.

24 Gustavsson M, Wilson MA, Mallard C, Rousset C, Johnston MV, Hagberg H: Global gene expression in the developing rat brain after hypoxic preconditioning: involvement of apoptotic mechanisms? Pediatr Res 2007;61: 444-450.

25 Hong F, Freeman ML, Liebler DC: Identification of sensor cysteines in human Keap1 modified by the cancer chemopreventive agent sulforaphane. Chem Res Toxicol 2005; 18:1917-1926.

26 Rahman I, Gilmour PS, Jimenez LA, MacNee $\mathrm{W}$ : Oxidative stress and TNF-alpha induce histone acetylation and NF-kappaB/AP-1 activation in alveolar epithelial cells: potential mechanism in gene transcription in lung inflammation. Mol Cell Biochem 2002;234-235:239-248.

27 El Gazzar M, Yoza BK, Chen X, Hu J, Hawkins GA, McCall CE: G9a and HP1 couple histone and DNA methylation to TNFalpha transcription silencing during endotoxin tolerance. J Biol Chem 2008;283:32198-32208.

28 Vakoc CR, Mandat SA, Olenchock BA, Blobel GA: Histone H3 lysine 9 methylation and HP1gamma are associated with transcription elongation through mammalian chromatin. Mol Cell 2005;19:381-391.

29 Kawai Y, Garduno L, Theodore M, Yang J, Arinze IJ: Acetylation-deacetylation of the transcription factor Nrf2 (nuclear factor erythroid 2-related factor 2) regulates its transcriptional activity and nucleocytoplasmic localization. J Biol Chem 2011;286:7629-7640.

30 Huang WC, Chen CC: Akt phosphorylation of p300 at Ser-1834 is essential for its histone acetyltransferase and transcriptional activity. Mol Cell Biol 2005;25:6592-6602.

- 31 Naidu S, Vijayan V, Santoso S, Kietzmann T, Immenschuh S: Inhibition and genetic deficiency of p38 MAPK up-regulates heme oxygenase-1 gene expression via Nrf2. J Immunol 2009;182:7048-7057.

32 Yu R, Chen C, Mo YY, Hebbar V, Owuor ED, Tan TH, Kong AN: Activation of mitogenactivated protein kinase pathways induces antioxidant response element-mediated gene expression via a Nrf2-dependent mechanism. J Biol Chem 2000;275:39907-39913.

33 Wang X, Zhu C, Qiu L, Hagberg H, Sandberg $\mathrm{M}$, Blomgren K: Activation of ERK1/2 after neonatal rat cerebral hypoxia-ischaemia. J Neurochem 2003;86:351-362. 